\title{
The Need for a Facility to Understand Volatile Ice Rheology
}

Caitlin Ahrens ${ }^{1}$ (332 N Arkansas Ave., Fayetteville, AR 72701, ca006@uark.edu)

Orkan Umurhan $^{2}$, Aaron Morrison ${ }^{3}$; Erica Nathan ${ }^{4}$

${ }^{1}$ Arkansas Center for Space and Planetary Sciences

${ }^{2}$ SETI Institute, NASA Ames Research Center

${ }^{3}$ University of Texas San Antonio

${ }^{4}$ Brown University

Endorsers: Christian Klimczak (University of Georgia); Patrick Matulka (Washington University in St. Louis); Chimira Andres (University of Western Ontario); Katherine Dzurilla (University of Arkansas); Al Emran (University of Arkansas)

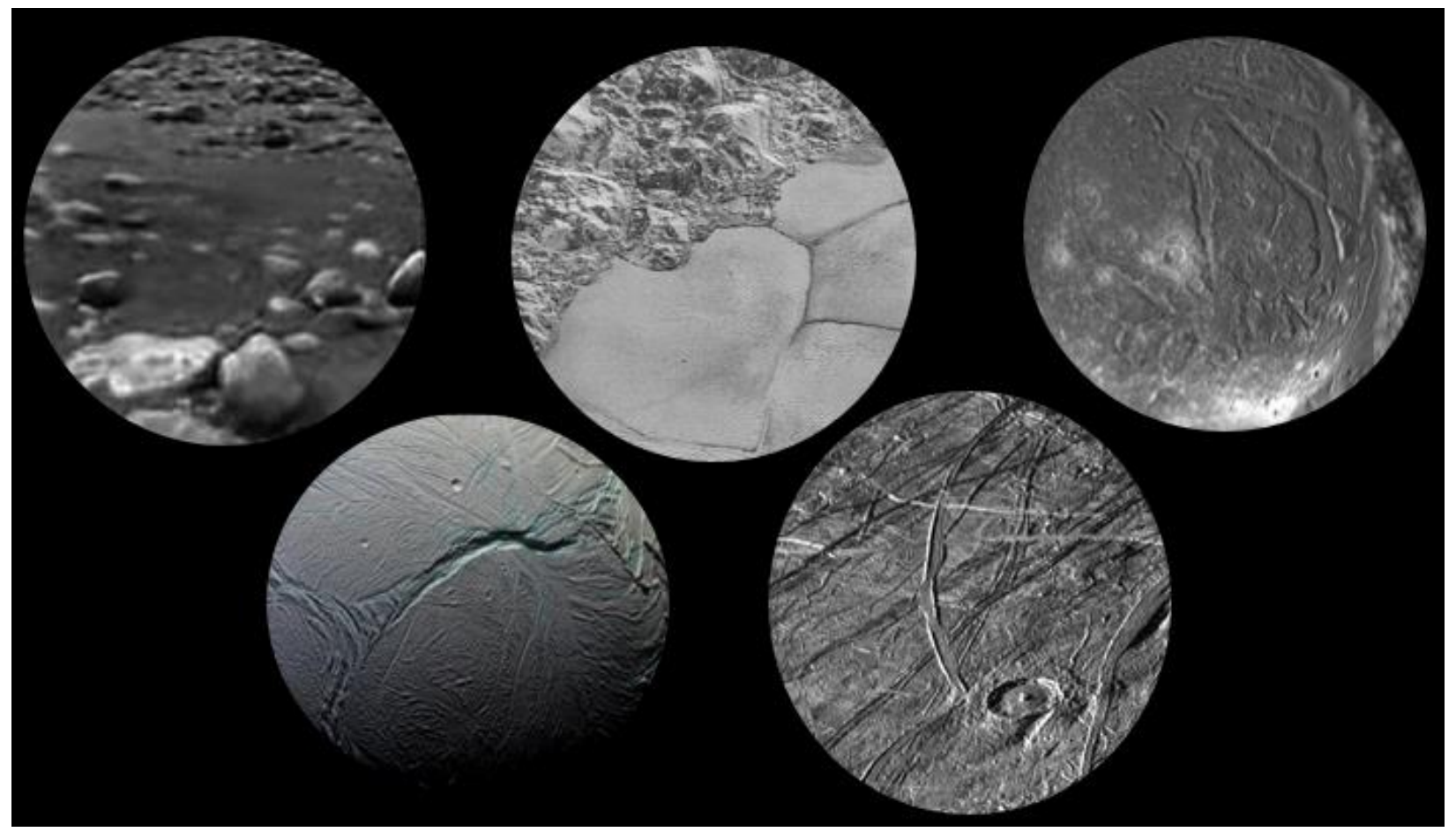




\section{Executive Summary}

The outer Solar System bodies, including satellites, dwarf planets, comets, and Kuiper Belt objects, consist of a variety of ices of volatile molecules and various complex hydrocarbons components. Laboratory measurements of physical properties of such materials generate key support for rheological dynamics and provide objectives of ground-based, orbital, and lander observations; instrument and mission design; and interpretation of retrieved data products. In some situations, models take the lead in characterizing these icy materials, especially where certain geophysical features, such as subsurface layering, cannot be directly observed. However, there are currently an insufficient number of laboratories equipped to measure the rheological and thermal properties of icy materials relative to the outer Solar System. The complexities of such ices, organics, and exotic mixtures thereof have been studied little in a laboratory setting due to challenging experimental constraints. We recommend increased support for such laboratory research of the rheological and thermal properties of icy planetary materials, especially being relevant to future space missions to observe such ices and extend our knowledge of these properties in a variety of low temperatures and strain rates.

\section{Science Rationale and Objectives}

We summarize in this white paper a collective effort of the outer planets science community in outlining the scientific need for a rheologic-specific laboratory and its direction for future NASA Discovery and Orbiter class mission studies, and the coming 2023 Decadal Survey to the National Research Council. This white paper addresses the components and motivations to the rheological and thermodynamic explorations of icy surface properties as a whole and basis for future experimental investigative studies. The following science objectives for volatile ice rheology in the laboratory are:

\section{Characterizing thermo-mechanical conditions}

(i) Range of low temperatures $(<100 \mathrm{~K})$

(ii) Amorphous and crystalline relationships

(iii) Volatile and organic mixtures

(iv) Ice phase transitions (e.g., solid-solid phase changes)

(v) Solid-liquid transitions/interactions

(vi) Grain size and porosity testing conditions

(vii) Mineralogical changes in ice growth (including co-crystals)

(viii) Sublimation pressures and processes

Strain and stress parametrization

(i) Stickiness of grains

(ii) Torsional stresses

(iii) Brittleness vs ductility of ice (pure and mixtures) 
(iv) Tensile, compressive, shear stress tests

Development of instrumentation

(i) Low-temperature, ultra-high vacuum systems

(ii) Reduction of ice stickiness/residues

(iii) Torsional, compressive, shear stress functions and monitoring

(iv) Brittleness/ductility monitoring and analysis

(v) Instrumentation for returned samples

The discussion is divided into three main sections, addressing fundamental questions as they pertain to the need for a volatile rheology laboratory facility:

-What planetary discoveries require laboratory investigation?

-What progress can be made in the next decade?

-What are the key challenges?

The role of experimental research in these physical properties is multifold. Ices synthesized in the laboratory setting are necessary for comparison to observational measurements and expand modeling objectives. A comprehensive table on specific science investigations benefiting from laboratory research is in Table 1.

Table 1: Mission observations, modeling, and laboratory approaches to key rheological-related questions. Adapted from Ahrens et al. (2018).

\begin{tabular}{|c|c|c|c|}
\hline Science Questions & $\begin{array}{l}\text { Ground-based, Fly- } \\
\text { by, Orbiter } \\
\text { Observation }\end{array}$ & Modeling Approach & $\begin{array}{l}\text { Laboratory } \\
\text { Measurement }\end{array}$ \\
\hline $\begin{array}{l}\text { What is the } \\
\text { composition and } \\
\text { structure of the } \\
\text { surface material? }\end{array}$ & $\begin{array}{l}\text { IR/VIS/UV } \\
\text { measurements } \\
\text { Density }\end{array}$ & $\begin{array}{l}\text { Volatile interactions } \\
\text { Regolith layering } \\
\text { Phase changes }\end{array}$ & $\begin{array}{l}\text { Thermophysical } \\
\text { (density, heat } \\
\text { capacity, latent heat); } \\
\text { Thermodynamic } \\
\text { (phase transitions, } \\
\text { pressure-volume, } \\
\text { equations of state); } \\
\text { Rheological (strain } \\
\text { rates, brittleness- } \\
\text { ductility, viscosity); } \\
\text { Dielectric parameters }\end{array}$ \\
\hline $\begin{array}{l}\text { What are the } \\
\text { radiative processes }\end{array}$ & $\begin{array}{l}\text { Temperature } \\
\text { Heat flux } \\
\text { IR/VIS/UV }\end{array}$ & $\begin{array}{l}\text { Radiative evolution } \\
\text { Thermal evolution }\end{array}$ & $\begin{array}{l}\text { Sublimation; } \\
\text { Phase stability; } \\
\text { Ion/photon radiation; }\end{array}$ \\
\hline
\end{tabular}




\begin{tabular}{|l|l|l|l|}
\hline $\begin{array}{l}\text { and surface } \\
\text { evolution? }\end{array}$ & & Heat capacity \\
\hline $\begin{array}{l}\text { What is the } \\
\text { relationship between } \\
\text { amorphous and } \\
\text { crystalline ices? }\end{array}$ & $\begin{array}{l}\text { Temperature } \\
\text { Heat flux } \\
\text { IR/VIS/UV }\end{array}$ & $\begin{array}{l}\text { Volatile stability } \\
\text { Phase changes }\end{array}$ & $\begin{array}{l}\text { Complex mixtures; } \\
\text { Sublimation; } \\
\text { Phase stability; } \\
\text { Thermodynamic } \\
\text { properties }\end{array}$ \\
\hline
\end{tabular}

\section{What Planetary Discoveries Require Laboratory Investigation?}

Here we display just a few examples of surface features observed on outer solar system icy bodies specific for the need of rheological and thermophysical laboratory investigations, with possible future experiments regarding each surface feature.

Miranda has probably one of the most complex surfaces of the main 5 satellites of Uranus. Miranda has some of the weirdest geologic structures across its surface. These relatively younger terrain units have not been observed elsewhere in the entire solar system. The interiors of this unit are marked by belts of differing albedo and outlined by parallel ridges- this unit is called "coronae" (Smith et al. 1986). Miranda also has chaotic fault blocks, fault scarps, and localized cryovolcanism, convection and thermal expansion (Schenk, 1991; Pappalardo et al., 1997; Hammond and Barr, 2014; Beddingfield et al. 2015)

Possible laboratory investigations toward understanding Miranda would include:

(i) Sliding ice block, frictional ice experiments

(ii) Compressional and shear stresses

(iii) Thermal expansions/relaxations

Triton, a water-ice world of Neptune, is considered to have a subsurface ocean and layers of volatile ices (nitrogen, methane) within the crust (Merlin et al. 2017; Hammond et a.. 2018). Being a captured Kuiper Belt object and a candidate ocean world, Triton is of interest for future Ocean Worlds objectives. Voyager 2's passage across the Neptunian system in 1989 captured images of streak-like features interpreted to be geysers (Duxbury and Brown 1997).

Possible laboratory investigations toward understanding Triton would include:

(i) Brittle-ductile transitions

(ii) Sublimation pressures

(iii) Cryovolcanic materials (e.g., viscosity) 
Pluto has a variety of interesting geologic environments as imaged by the NASA New Horizons fly-by mission. Of particular interest for rheological experimental investigation is the glacial flow and large bladed terrain (Umurhan et al. 2017). Glacial flow of volatile ices and floating blocks of water ice on the slowly convecting Sputnik Planitia give insight to the glacial deposition and debris, sliding mechanics, and channel valley flow of complex, intimate icy mixtures (Schenk et al. 2018). The bladed terrain (Tartarus Dorsa) on the eastern close-encounter hemisphere of Pluto display massive, angled, pointed structures as a possible result of sublimation processes (Moores et al. 2017). However, the deformation and basal stresses, and the ice densities involved are still to be investigated.

Possible laboratory investigations toward understanding Pluto would include:

(i) Frictional ice experiments

(ii) Deformational stresses

(iii) Tensile strength (e.g., impact cratering experiments)

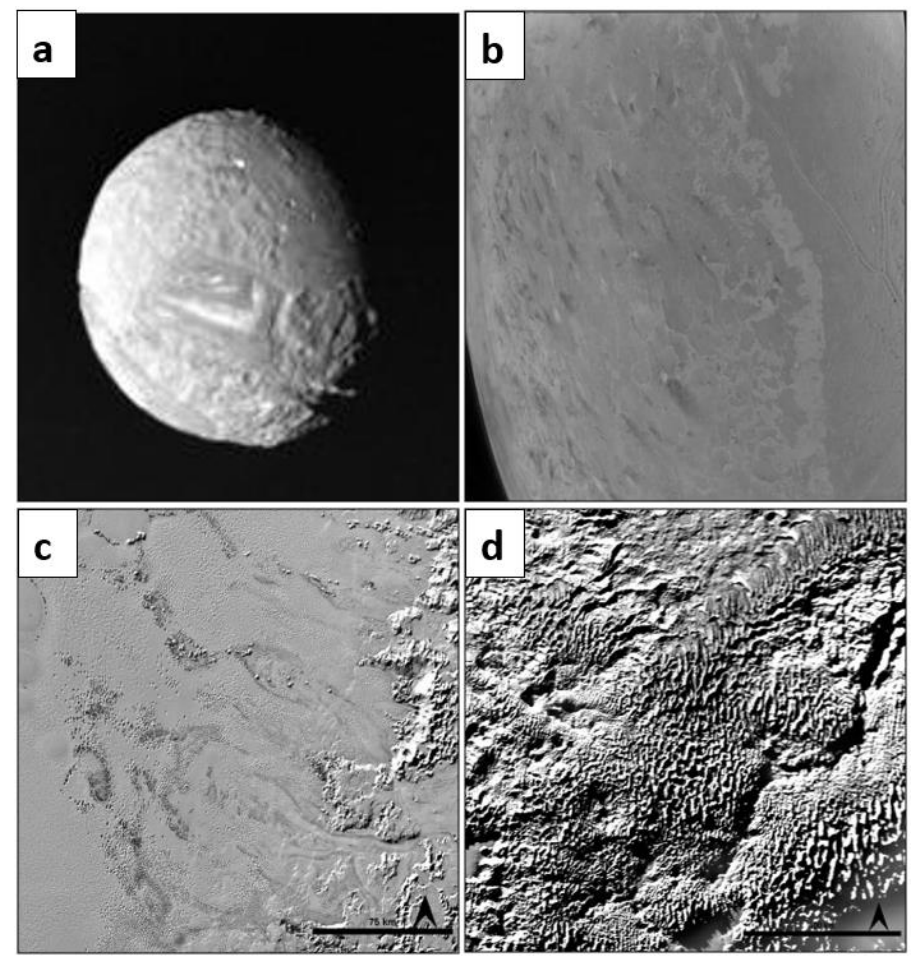

Figure 1: a) Miranda imaged by Voyager 2. Inverness Corona is in center. (RING OBS ID: U_IMG_VG2_ISS_2684630_W); b) Triton dark-streaked geysers imaged by Voyager 2. (OPUS ID: vg-iss-2-n-c1139503); c) portion of eastern Sputnik Planitia shores displaying glacial flows imaged by New Horizons. Scale bar at $75 \mathrm{~km}$; d) Tartarus Dorsa bladed terrain as imaged by New Horizons. Scale bare at $100 \mathrm{~km}$. 
Returned samples are also prime materials for advancing experimental rheological research. Of particular interest are those from the Moon (white paper by Jolliff et al. 2020); asteroids, such as the OSIRIS-REx mission (Lauretta et al. 2017); and the possibility of other surfaces, such as Titan (see White paper by Landis et al. 2020) and other ocean worlds (see white paper by Hudson et al. 2020). Of particular interest with such sample returns would be the expanded knowledge of material science across the solar system. Such knowledge can then be explored with synthetic recreations of the sample for reproduction purposes (simulants) for laboratory purposes (Smith et al. 2010; Metzger et al. 2019).

Possible laboratory investigations toward understanding sample return materials would include:

(i) Regolith grain sizes and cohesiveness

(ii) Thermal properties (i.e., dielectric, heat capacity)

(iii) Recreation of material for production of simulants

\section{What Progress Can Be Made in the Next Decade?}

A number of future planetary missions are in the works. However, certain instruments must be advocated to those missions that will be crucial to the experimental research of icy materials. These include the mapping of icy compositions, radar, solar irradiation readings, and temperature-pressure measurements.

To supplement these observations, advances in modeling capabilities can further assist in ice phases and interactions of such ices. Observing geomorphologic regions will help to improve model predictions regarding icy surfaces, interior-surface interactions, and possibly seasonal (or tidal) interactions. Dynamical modeling to look at the influence of tides on icy satellites and their surfaces would also be constructive as predictions prior to a mission re-visiting the system.

Many of these questions are not exclusive to the surface process community alone and answering them will require an interdisciplinary and collaborative approach. For example, interpreting the discovery of glaciers on Pluto, is a problem of rheology, glaciology, and structural geology.

\section{What are the Key Challenges?}

Laboratory studies include, but are not limited to, atmospheric composition, chemistry, surface composition, geology, geophysics, and planetary interiors. The range of experiments range from spectroscopy to compositional studies, chemical and physical reactions, and parameterizing possible scenarios in low temperatures.

A few key challenges to further our analysis of the rheology and thermal properties of the outer Solar System include measurements of planetary materials (chemistry, mineralogy, etc.) that requires controlled sampling, testing and verification of results, reproducibility of measurements, 
and interpretation of results. Included in laboratory challenges are listed (though not limited) below (from Ahrens et al. 2018):

(i) Variety of extreme conditions: While extrapolation of certain ices and gases are used for modeling purposes, this could prove poor in predictions for experimentation. Most outer Solar System planetary bodies are in microbar pressures and $<100 \mathrm{~K}$ temperatures. These conditions require custom-made facilities.

(ii) Phase diagram verification: mixtures of ices, gases, and sometimes more complicated chemistries (i.e. hydrocarbons) for compositional studies and phase changes are not well documented. This could cause verification of varying mixtures and conditions to deem difficult. As a result, a database of such thermophysical properties must be a priority.

(iii) Time and funding: From sample preparation and instrument calibration for extreme low temperature conditions to the actual analysis takes time, and often, expenses. This could include specific personnel to handle certain equipment, shipment of gases, or software support.

\section{Recommendations}

Based on the above details, we recommend that the Outer Planets Assessment Group (OPAG), Committee on Astrobiology and Planetary Sciences (CAPS), and Decadal Survey review panel support the following points (in no particular rank):

(a) Establishment of a designated facility for the purpose of volatile ice rheological studies to enhance and improve current experimental knowledge.

(b) Improvement of coordination between laboratories working on outer solar system experiments and simulations for the improvement of networking and understanding of icy material behavior.

(c) Establishment of a database for sharing funded laboratory results (e.g. Planetary Data System).

(d) Support the role of experimental research as a key component of planetary exploration.

(e) Increased inclusion and representation from the laboratory research community within respective professional societies and assessment groups.

(f) Increased provisional funding inside mission proposals to support laboratory work dedicated to support specific mission instrumentation testing and science planning development requirements. 


\section{References}

Ahrens, C., et al. (2018) Recent experiments and motivations of simulated Pluto experiments. Space Sci. Rev., 214 (8), 24 pp.

Beddingfield, C., Burr, D., Emery, J. (2015) Fault geometries on Uranus' satellite Miranda: Implications for internal structure and heat flow. Icarus 247, 35-52.

Duxbury, N., and Brown, R., (1997) The role of an internal heat source for the eruptive plumes on Triton. Icarus, 125 (1), 83-93.

Hammond, N., Barr, A. (2014) Global resurfacing of Uranus's moon Miranda by convection. Geology, 42 (11), 931-934.

Hammond et al. (2018) Compaction and melt transport in ammonia-rich ice shells: Implications for the evolution of Triton. JGR: Planets, 123 (12), 3105-3118.

Hudson, R., et al. (2020) Lab research supporting Ocean Worlds exploration. NAS white paper submitted to the Planetary Science decadal survey committee.

Jolliff, B., et al. (2020) Sample return from the South Pole - Aitken Basin. NAS white paper submitted to the Planetary Science decadal survey committee.

Landis, G., et al. (2020) A proposed sample return from Titan. NAS white paper submitted to the Planetary Science decadal survey committee.

Lauretta, D., et al. (2017) OSIRIS-REx: Sample return from asteroid (101955) Bennu. Space Sci. Rev. 212, 925-984.

Merlin et al. (2017) Physical constraints on the ices present on Triton's surface. 49 AAS DPS meeting, Abstract 214.20.

Metzger, P., et al. (2019) Measuring the fidelity of asteroid regolith and cobble simulants. Icarus, $321,632-646$.

Moores, J. et al. (2017) Penitentes as the origin of the bladed terrain of Tartarus Dorsa on Pluto. Nature, 541 (7636), 188-190.

Pappalardo, R., Reynolds, S., Greeley, R. (1997) Extensional tilt blocks on Miranda: Evidence for an upwelling origin of Arden Corona. JGR: Planets, 102 (E6), 13369-13379.

Schenk, P. (1991) Fluid volcanism on Miranda and Ariel: flow morphology and composition. JGR, 96, 1887-906.

Schenk, P., et al. (2018) Basins, fractures and volcanoes: Global cartography and topography of Pluto from New Horizons. Icarus, 314, 400-433.

Smith, B., et al. (1986) Voyager 2 in the Uranian system: imaging science results. Science, 233, 43-64.

Smith, M., et al. (2010) Structural determination of Titan aerosol laboratory simulants. $42^{\text {nd }}$ AAS DPS Meeting, Abstract 1069.

Umurhan, O. et al. (2017) Modeling glacial flow on and onto Pluto's Sputnik Planitia. Icarus, 287, 301-319. 